CDD: 401

\title{
Book review: ALMOG, J. Referential Mechanics: Direct Reference and the Foundations of Semantics (Oxford University Press, 2014)
}

\section{Filipe Martone}

\author{
University of Campinas - Philosophy \\ Rua Cora Coralina 100 Campinas \\ São Paulo, Brazil \\ 13083-896 \\ filipemartone@gmail.com
}

Received: 25.07.2016; Accepted: 26.07.2016

DOI: http://dx.doi.org/10.1590/0100-6045.2016.V39N2.FM

\begin{abstract}
In this review I discuss Joseph Almog's book "Referential Mechanics". The book discusses direct reference as conceived by three of its founding fathers, Kripke, Kaplan and Donnellan, and introduces Almog's ambitious project of providing a referential semantics to all subject-phrases. I offer a brief overview of its four chapters and point out some of their virtues and shortcomings.
\end{abstract}

Keywords: direct reference, semantics, philosophy of language, foundational semantics

The work of Ruth Barcan Marcus, Saul Kripke, Keith Donnellan, David Kaplan and others started a revolution in philosophy of language. The so-called direct reference theory, or simply referentialism, dealt a powerful blow to the then prevailing Fregean spirit of theories of meaning and reference, and it deeply affected the way we think about these topics. But saying what the core ideas of direct reference really are is not as easy as one might think. Direct reference theorists often disagree about what seems to be very basic issues: Are there singular propositions, and do we need them? If descriptivism is false, what are the mechanisms of reference determination? What are the consequences (if any) of holding a referentialist semantics to our views on cognition? Should referentialists be worried about Frege's Puzzle? In a time in which debates in philosophy of language can get highly sophisticated, it is easy to get lost amidst technical arguments and overlook these fundamental 
questions. Joseph Almog's new book, Referential Mechanics: Direct Reference and the Foundations of Semantics, is an attempt to look past the technicalities of this "quantum mechanics of words" (p. xvii) and to engage with those questions directly.

Almog has three aims in this essay. First, he wants to understand what direct reference is really about. To do so, he dissects the versions of direct reference offered by three of its founding fathers - Kripke, Kaplan and Donnellan - and examines their foundations and their consequences for philosophy of mind and metaphysics. Second, he tries to show that two puzzles that supposedly threaten direct reference - the puzzle of empty names and Frege's Puzzle - are not puzzles at all. Third, and finally, he introduces his project of unifying the semantics of all subject-phrases under the referentialist framework: instead of modelling paradigmatically referential terms such as proper names after denoting phrases (e.g. "every philosopher" or "most philosophers"), as Montague and his followers did, Almog wants to treat denoting phrases as genuinely referential terms. The challenge of integrating referential semantics into global semantics is what he called the Russell-ParteeKaplan challenge. But Referential Mechanics only sets up the stage for a full answer to this challenge. A detailed account will be given in a companion piece, not yet published.

The book is divided into four chapters. The first three deal with direct reference as conceived by Kripke, Kaplan and Donnellan, and the fourth wrestles with the puzzles of empty names and cognitive significance (a.k.a. Frege's Puzzle) and begins to work on an answer to the Russell-Partee-Kaplan challenge. In what follows I will offer a brief overview of the chapters, pointing out some of their virtues as well as their shortcomings.

In the first chapter, Almog offers an interesting reading of Kripke's Naming and Necessity (henceforth NN) and introduces some of the key notions of his essay. He claims that there is a fundamental tension between two very different conceptions of semantics in NN. On the one hand, there is what he calls the designation model theory for natural language. This model is what we get from Lecture I. On this model, direct reference is cashed out in terms of rigid designation. On the other hand, there is the historical referential semantics, found mainly in Lecture III. The distinction between these two conceptions of semantics "touches the very fulcrum" of Almog's essay (p. 15). According to him, the designation model suffers from a fundamental defect; it completely 
misconceives what semantics truly is (or should be): a descriptively correct account of the actual workings of a natural language, and not an adequate but mere representation of such workings (p. 16).

The main problem with the designation model, Almog argues, is the following. In this model, expressions such as names, definite descriptions, sentences and predicates are all unified under the fundamental semantic relation of designation. This model of semantics, therefore, sees no categorical difference between proper names and definite descriptions. In other terms, even though names are rigid designators whereas descriptions are not, both are connected to their designata via the same kind of semantic relation. However, designation is a "stipulated relation designed for a language with uninterpreted symbols: individual constants, variables, predicates (...)" (p. 15). Thus, designation is not, Almog claims, the real semantic relation that obtains in an actual existing language like English. Taking it to be so creates an insolvable puzzle, namely, the puzzle of reference determination: descriptions pick out their designata via satisfaction or fit, but how do names pick out their designata if they are not disguised descriptions, as Kripke so convincingly argued? How does the designation relation obtain for proper names? This model of semantics has no answer to give.

The historical referential semantics, on the other hand, gets it right. There is no puzzle of reference determination to be solved. The names we use are already loaded with their referents. We, consumers of language, do not have to "reach out" to them; they come to us through their names via causal/communication chains. The puzzle of reference determination arises only we if take designation to be the fundamental semantic relation obtaining in actual languages. Once we see that it is not, the puzzle disappears. Almog calls this shift from an "inside-out" (designation) to an "outside-in" (historical referential) mechanism of reference the "flow diagram reversal": we refer with a name not because we designate an object, but because the object itself is already connected to the name we are using. This "flow diagram reversal", he claims, is present in the works of Kripke, Kaplan and Donnellan, and it is the key idea of direct reference.

Almog's notions of "outside-in" mechanisms of reference and of "flow diagram reversal" are quite interesting, and they neatly capture some of the main lessons of direct reference. However, he makes some rather cryptic remarks about perceiving an object through its name. More precisely, he claims 
that the reversal account of reference also extends "the classically qualitatively understood perception" (p. 29), meaning that "we perceive (remote) objects by means of loaded names" (p. 30). He offers some details of this view in footnote 19 , but they are not really illuminating. What he seems to be suggesting is that public names put us in some sort of "acquaintance" relation with their bearers, allowing us to cognize them through language. This is a respectable idea which has many advocates nowadays. Yet, to say that this kind of relation extends perception per se seems to stretch the notion of perception beyond plausibility.

The second chapter is devoted to Kaplan's account of direct reference in terms of singular propositions. For Kaplan, for a term to be directly referential is for it to make a special kind of contribution - its referent - to the proposition expressed by the sentence that contains it. This kind of proposition is called 'singular proposition'. Almog stresses how distinct Kaplan's approach to direct reference is when compared to Kripke's designation model. That model makes no use of propositions; its key semantic unit is the pre-sentential subject (the designator). Kaplan's direct reference semantics, on the other hand, takes propositions as its key semantic unit, and as the objects of assertion and thought. Almog argues that this leads to several problems, and these problems show that singular propositions fail to capture what direct reference is really about.

One interesting aspect of this chapter is that it tries to distinguish between what are legitimate criticisms of singular propositions and what are not. Almog vehemently dismisses the claims that the doctrine of singular propositions (and direct reference in general) is committed to a particular view about modal haecceitism and about the informativeness of identity statements (i.e. Frege's Puzzle). Singular propositions and direct reference, he argues, are merely semantic notions. As he puts it: "There are no entailments from direct reference theory proper regarding either modal or attitudinal questions" (p. 48; italics in the original). What are legitimate criticisms of singular propositions, on the other hand, are the well-known objections of negative existentials with empty names and of reference to past objects.

What is a bit strange in this chapter is Almog's claim that the failure of singular propositions to correctly classify apparently contradictory cognizers like Kripke's Pierre and to capture phenomena of cognitive dynamics adequately "leads to the breakdown of the apparatus of proposition and content" (p. 56). If singular propositions are neutral regarding "attitudinal questions", as he 
says, why are arguments from cognitive dynamics and propositional attitudes wielded against them? Why is Frege's Puzzle not a problem to singular propositions while Kripke's Pierre is? In fact, Kripke's Pierre seems to be an instance of Frege's Puzzle, so it is not at all clear why singular propositions are threatened by the first and not by the latter. Almog is also quite vague when it comes to his alternative to singular propositions. He talks of "object-loaded names" coming to us and of sentences with empty names being true precisely "because there is no proposition" corresponding to them. These remarks are somewhat obscure. He says, for instance, that "On this idea of the re-ferent coming to us late users, there is no mystery about why you and I can and do refer now to the long gone Aristotle by using now the (Aristotle-loaded) name 'Aristotle', just as we see a long dead star by being impacted now by light from it" (p. 52). There is plenty of mystery there to me, however. If the object no longer exists, how come it is loaded into the name? How do we "refer back" to Aristotle and cognize him, as Almog often says, if Aristotle does not exist anymore? Almog probably means that there is a causal connection between Aristotle, "Aristotle" and us, but that is not a complete explanation of what sort of thing we are in fact cognizing and referring to when using a name. Besides, it is also not obvious why his objections to singular propositions do not apply to his own view, since objects themselves play a crucial role in his semantics, as they do in Kaplan's account.

The third chapter is by far the most compelling part of Almog's book. It discusses Donnellan's idea of referential uses of expressions and its connection to having an object in mind. Almog believes that these two ideas are the key ideas not only of direct reference, but of semantics in general. In discussing them he hopes to show that semantics should be conceived not as a branch of model theory (as Montague held), but as branch of cognitive psychology (p. 63).

Almog claims that Donnellan's insight about having an object in mind in fact explains direct reference: direct reference is direct not because of conventional rules of language, but because it is linked to a certain cognitive mechanism of grasping worldly objects. This mechanism is captured by the "flow diagram reversal": we do not have to reach out to those worldly objects; our minds, by natural processes, enter in relations with those objects, and they "make their way" into our cognition. It is not necessary to select which object we are thinking of and then look for it in the world. In fact, Almog argues that it is a mistake to understand Donnellan's idea of having an object in mind as 
internally selecting some individual or other; the object we have in mind is determined by causal/historical processes. It is because we have objects in mind in this way that we can directly refer to it.

Almog argues that the process of referential use is divided into three different stages. First, there is the fixing. My mind enters in some relation with an object by an outside-in mechanism, which makes that precise object the object of my cognition. Second, there is the characterization. After the object is determined by this process, I can predicate things of it. Almog stresses that, because the object is already fixed, it does not matter if I apply false predicates; my thought is about it even in cases of gross mischaracterization. This is precisely what happens in Donnellan's cases. Third, there is the communication. After (1) the object is fixed and (2) I form some beliefs about it, I can (3) go on to express these beliefs through language. To do that, I use whatever expression I think will help to direct the audience's attention to the object I am already thinking of. As Almog puts it: "I am trying to co-focus you, make you have in mind what I already have in mind" (p. 69). This is why, for him, referential uses are not in any way restricted to definite descriptions. Any kind of singular terms, and even expressions like "someone", can be used referentially: these terms do not determine the reference; they are used merely as aids to communication.

With this Almog hopes to show why Kripke's claim that Donnellan's cases are cases of speaker reference and not of semantic reference is mistaken. He believes that this distinction is preposterous; they are all as semantic as it gets. However, I do not see why this conclusion follows from his arguments. His proposal might in fact account for referential uses, but it is not clear why it also entails that there is no such thing as the semantic referent of an expression in a particular use. Even if our cognition is hooked to objects by non-conventional processes (which is a very plausible idea), it does not follow that this sort of having in mind completely overrides the conventional reference of the expressions used. In other words, I do not see why it is incompatible to hold this "flow diagram reversal" for thought and the speaker/semantic reference distinction at the same time. In short, more arguments are required to show why Donnellan's "cognitive mechanics" entails the collapse of the speaker/semantic referent distinction.

In sum, Almog seems to be proposing some sort of externalist-subjectivist semantics in this chapter. It is externalist because external objects themselves make their way into our cognition, and the meanings of the expressions we use 
(if we are allowed to talk about meanings in Almog's framework) are external objects and properties. Yet, it is subjectivist because what matters for giving the content (again, if such talk is plausible here) of the expressions we use are not community-wide conventions, but individual speakers in particular occasions. This is not, of course, to deny the role of conventions, but what ultimately determines the content/referents of our expressions is what the speakers have in mind when they use these expressions. This combination of two apparently opposed views about language is certainly interesting and it deserves further discussion.

The fourth and last chapter is concerned with Frege's Puzzle and the puzzle of empty names, and it introduces the discussion of the Russell-ParteeKaplan challenge. Almog argues that, if we take Donnellan's approach seriously, both puzzles disappear. In fact, they turn out to be uninteresting consequences of this model of semantics (p. 91). The first observation he makes is that no speaker is omniscient regarding the semantic history of all names she uses. If this is right, informative identity statements are to be expected precisely because knowing that two names lead back to the same object can only be known a posteriori. Informativeness, Almog claims, is a relational feature, and it arises from the interaction between the information a speaker has in the head and the relevant sentence. If this in-the-head information is sufficient to settle the truth of the sentence, it will be trivial; if not, it will be informative. Second, the puzzle of empty names disappears because what makes sentences containing such names true are not objectinvolving propositions; what makes them true is the history of the name that leads back to failed baptisms.

Almog puts the Russell-Partee-Kaplan challenge as follows: "Can we or can't we generalize the reference-only semantics to all nominals?" (p. 98). The problem arises from a dilemma about the visible grammatical form of subjectpredicate sentences and their semantic forms. Subject-predicate sentences apparently work in the same way: they introduce objects for subsequent predication. Some terms, like proper names and indexicals, seem to fit well in this intuitive definition. Their visible grammar is a reliable guide to their semantic (or logical) forms. The problem is that some subject-predicate sentences, like "most philosophers are wise" or "some linguists sing", do not seem to refer to any object. How should we deal with this? Russell, as Almog says, held that not all nominals function semantically in the same way: some 
refer, some denote. Montague, on the other hand, wanted to keep the link between visible grammar and semantics, but to do that he opted to treat referential terms as he treated other denoting nominals: visible grammar reflects semantic form insofar as all subject terms denote and not refer. Almog, however, wants to keep the semantics of all nominals referential and the visible grammar intact. So, he needs to explain what sort of reference expressions like "most philosophers" and "many linguists" have and how we should treat subject-predicate sentences containing them.

Almog claims that all nominals can be used either referentially or attributively. In the first case, they merely communicate an already made reference to an object, i.e., they externalize what I have in mind; in the second, they originate reference. This is a genuine semantic "ambiguity" of nominals, and not a mere pragmatic phenomenon. Both uses, however, necessarily involve reference to some worldly entity. As for their reference, Almog claims that nominals like "every musician" and "two philosophers" pick out kinds or "pluralities" in the world (p. 111). In this way, they do not denote, but genuinely refer to these entities. He closes the chapter by considering some challenges and further objectives (to be discussed in another essay): he wants to extend his account beyond subject-predicate sentences, apply it to the logical relation of consequence and to understand what is to cognize the world by using ordinary language - all of this following the general guidelines of keeping the semantics referential and the superficial grammar untouched.

Referential Mechanics is an engaging and provocative book, even though its difficult subject and the many topics it discusses would probably receive a better treatment in a longer and more detailed essay. Its occasional lack of clarity and insufficient argumentation can get frustrating sometimes. However, this does not diminish its many merits. The essay advances unorthodox theses and offers interesting takes on three of the most important versions of direct reference. Anyone interested in semantics, foundational semantics and in the history of analytic philosophy should definitely study it.

\section{Referencias}

Almog, Joseph. Referential Mechanics: Direct Reference and the Foundations of Semantics. Oxford: Oxford University Press, 2014. 


\section{Erratum:}

DOI: http://dx.doi.org/10.1590/0100-6045.2016.V39N2.FME

In the article "Referential Mechanics: Direct Reference and the Foundations of Semantics" with DOI: http://dx.doi.org/10.1590/0100-

6045.2016.V39N2.FM, published in MANUSCRITO, 39.2, 133 a 140 , on 133, where it says

"Referential Mechanics: Direct Reference and the Foundations of Semantics"

one should read

"Book Review: Joseph Almog, Referential Mechanics: Direct Reference and the Foundations of Semantics (Oxford University Press, 2014)"

Although this article is included in the present volume, it appeared earlier in the modality Ahead of Print. 